\title{
BMJ Global Health Parent-level barriers to uptake of childhood vaccination: a global overview of systematic reviews
}

\author{
Jessica Kaufman, ${ }^{1,2}$ Jane Tuckerman,, ${ }^{1,2}$ Carissa Bonner, ${ }^{3}$ David N Durrheim, ${ }^{4,5}$ \\ Daniel Costa, ${ }^{3}$ Lyndal Trevena, ${ }^{3}$ Susan Thomas, ${ }^{4,5}$ Margie Danchin ${ }^{1,2}$
}

To cite: Kaufman J,

Tuckerman J, Bonner C, et al. Parent-level barriers to uptake of childhood vaccination: a global overview of systematic reviews. BMJ Global Health 2021;6:e006860. doi:10.1136/ bmjgh-2021-006860

Handling editor Seye Abimbola

- Additional supplemental material is published online only. To view, please visit the journal online (http://dx.doi.org/10. 1136/bmjgh-2021-006860)

JK and JT are joint first authors.

Received 9 July 2021

Accepted 9 September 2021

Check for updates

C Author(s) (or their employer(s)) 2021. Re-use permitted under CC BY-NC. No commercial re-use. See rights and permissions. Published by BMJ.

For numbered affiliations see end of article.

Correspondence to

Jessica Kaufman

jess.kaufman@mcri.edu.au

\section{ABSTRACT}

Introduction Understanding barriers to childhood vaccination is crucial to inform effective interventions for maximising uptake. Published systematic reviews include different primary studies, producing varying lists of barriers. To make sense of this diverse body of literature, a comprehensive level of summary and synthesis is necessary. This overview of systematic reviews maps all potential parent-level barriers to childhood vaccination identified in systematic reviews. It synthesises these into a conceptual framework to inform development of a vaccine barriers assessment tool.

Methods We applied Joanna Briggs methodology, searching the Epistemonikos review database and reference lists of included reviews to June 2020. Systematic reviews of qualitative or quantitative data on parent-level barriers to routine vaccination in preschoolaged children were included. Reviews addressing influenza, reporting non-modifiable determinants or reporting barriers not relevant to parents were excluded. Where possible, we extracted review details, barrier descriptions and the number, setting and design of primary studies. Two authors independently screened search results and inductively coded barrier descriptions.

Results We screened 464 papers, identifying 30 relevant reviews with minimal overlap. Fourteen reviews included qualitative and quantitative primary studies, seven included quantitative and seven included qualitative studies only. Two did not report included study designs. Two-thirds of reviews $(n=20 ; 67 \%)$ only included primary studies from high-income countries. We extracted 573 barrier descriptions and inductively coded these into 64 unique barriers in six overarching categories: (1) Access, (2) Clinic or Health System Barriers, (3) Concerns and Beliefs, (4) Health Perceptions and Experiences, (5) Knowledge and Information and (6) Social or Family Influence.

Conclusions A global overview of systematic reviews of parent-level barriers to childhood vaccine uptake identified 64 barriers to inform development of a new comprehensive survey instrument. This instrument will assess both access and acceptance barriers to more accurately diagnose the reasons for under-vaccination in children in different settings.

\section{INTRODUCTION}

The public health achievements of vaccines are threatened by those parents who decline,

\section{WHAT IS ALREADY KNOWN?}

$\Rightarrow$ The barriers to childhood vaccination include both access and acceptance barriers.

$\Rightarrow$ Strategies to improve childhood vaccination coverage often do not address all relevant key barriers to uptake and are therefore less effective and more costly.

$\Rightarrow$ There are no validated survey instruments that can measure both access and acceptance barriers to enable accurate interventions to be developed and tailored to different populations or settings.

\section{WHAT ARE THE NEW FINDINGS?}

$\Rightarrow$ Both qualitative and quantitative data identified key barriers to childhood vaccination from the parents' perspective, but no single review included all potential barriers.

$\Rightarrow$ Six categories of barriers were identified, encompassing barriers related to access, clinic or health system, concerns and beliefs, health perceptions and experiences, knowledge and information and social or family influence.

$\Rightarrow$ Two thirds of systematic reviews of barriers to childhood vaccination were from high-income countries only.

\section{WHAT DO THE NEW FINDINGS IMPLY?}

$\Rightarrow$ Surveys can only identify and address barriers they are designed to detect, so measurement instruments need to be developed and validated based on a comprehensive understanding of all potential barriers.

$\Rightarrow$ These findings will inform the development of a new tool in Australia and New Zealand to measure both access and acceptance barriers in high-income, middle-income and low-income settings.

$\Rightarrow$ Future systematic reviews of barriers should identify the primary studies contributing data on each barrier and report evidence of association between barriers and vaccination rates.

delay or are unable to access vaccines for their children. Globally, we have seen the devastating effects of vaccine-preventable disease outbreaks, such as the $130 \%$ increase in global measles deaths between 2016 and 2019. ${ }^{1}$ The 
COVID-19 vaccine rollout has further accentuated the prevailing challenges of reaching targeted communities with routine childhood vaccines. ${ }^{2}$

Under-vaccination and non-vaccination is due to a context-specific combination of barriers associated with both acceptance and access. Much attention is paid to acceptance, particularly since the WHO named vaccine hesitancy as one the 10 greatest threats to global health. ${ }^{3}$ However, countries can still have suboptimal childhood vaccination coverage ${ }^{45}$ despite high levels of vaccine confidence and demand, highlighting that access barriers are an often overlooked and important issue to identify and address. ${ }^{6}$ Any factor that inhibits vaccine uptake can be considered a barrier, whether it is psychological or physical. Some barriers exist at a health system level, while others are experienced or perceived at the individual or parent level.

Survey instruments are often used to identify barriers in different populations or settings to develop, target and evaluate interventions to improve vaccine uptake. However, a survey is inherently limited by what it is designed to measure and the response options available. Barriers that are not included in the instrument may go undetected and therefore unaddressed by interventions, leading to costly and ineffective strategies. Surveys may be supplemented with or informed by qualitative research to explore the experiences of participants, including both providers and parents, to unearth unique barriers. However, depending on the setting, these barriers may not be widespread or generalisable.

Published systematic reviews have synthesised data on barriers to childhood vaccination from different, sometimes overlapping, selections of quantitative and qualitative primary studies. However, no overview has ever synthesised these systematic reviews to comprehensively summarise the global literature on barriers identified through both data collection methods, from the parents' perspective.

In this overview of systematic reviews, we identify and conceptually map all potential parent-level barriers to childhood vaccination identified in published systematic reviews of qualitative and quantitative primary studies.

\section{METHODS}

We applied Joanna Briggs methods for overviews (or 'umbrella reviews'), ${ }^{7}$ which we defined a priori. We published the protocol online prior to undertaking the screening and data extraction process. ${ }^{8}$ Reporting follows the Preferred Reporting Items for Systematic Reviews and Meta-Analyses (PRISMA) reporting checklist (see online supplemental materials). ${ }^{9}$

\section{Review questions}

The primary review question was:

- What are the barriers to vaccination of preschool-aged children (under age 6) experienced or perceived by parents?
The secondary questions were:

- Do the barriers differ by the primary research methods that identified them?

- What barriers are identified in high-income countries (HIC), low-income or middle-income countries (LMIC)?

- What evidence of association between specific barriers and vaccine uptake is available?

\section{Selection criteria}

We included systematic reviews of quantitative, qualitative or mixed-methods primary studies that explored reasons for non-vaccination, barriers to vaccination or factors associated with uptake of childhood vaccines. We defined 'childhood vaccination' to include all routine vaccines given to preschool-aged children (under 6 years). Reviews were eligible for inclusion if they discussed parent-level barriers, that is, barriers experienced by and relevant to parents directly. Reviews with mixed-age populations were included if one or more primary studies focused on vaccination for preschool-aged children, but data were only extracted on barriers explicitly related to childhood vaccination. No publication language or date restrictions were applied.

\section{Exclusion criteria}

- Reviews focusing solely on adolescent or adult vaccination (eg, human papillomavirus vaccines)

- Reviews focusing solely on non-modifiable determinants (eg, ethnicity, socioeconomic status and gender) or barriers not directly relevant to parents (eg, healthcare provider training).

- Non-systematic reviews, that is, literature reviews that did not describe the search strategy and selection criteria for primary studies, and/or did not report the number and references of included studies

- Reviews focusing solely on intervention studies

- Reviews focusing solely on seasonal or pandemic influenza vaccines.

\section{Search and screening}

We searched for systematic reviews using the Epistemonikos database. Epistemonikos regularly indexes systematic reviews from a range of other databases, including the Cochrane Database of Systematic Reviews (CDSR), Pubmed, Embase, CINAHL (The Cumulative Index to Nursing and Allied Health Literature), Psycinfo, LILACS (Literatura Latinoamericana y del Caribe en Ciencias de la Salud), Database of Abstracts of Reviews of Effects (DARE), The Campbell Collaboration online library, JBI Database of Systematic Reviews and Implementation and EPPI-Centre Evidence Library. We did not place any date or language restrictions on the search. We also checked the reference lists of included reviews. Our search strategy is included in the online supplemental files. The search was conducted in June 2020.

Using Covidence, ${ }^{10}$ two members of the research team (JK and JT) independently screened all titles and abstracts 
to identify potentially eligible reviews. Full-text reviews were examined independently to determine inclusion. Disagreements were resolved through discussion with additional authors (CB and MD). Reasons for exclusion of all reviews examined in full text were recorded (see online supplemental files).

\section{Data extraction}

Using a pilot-tested data extraction form, one author extracted all data, with a second author checking and verifying data from a random sample of five included reviews. The following data were extracted for all included reviews:

- Publication date

- Stated review focus.

- Vaccines of interest (eg, all routine childhood vaccines; specific vaccines)

- Stated selection criteria for primary studies (including whether quality of primary studies was appraised)

- Number and type of primary studies (eg, qualitative, quantitative mixed methods).

- Country and country income level of primary studies (as defined by the World Bank)

- Summary of primary study geographic settings, if stated (eg, urban/rural, international/national/ subnational/local)

- Summary of primary study health system setting, if stated (public/private, community health, hospital-based).

We extracted the following specific data for all reported barriers noted or perceived by parents to be related to vaccine behaviour or uptake:

- Description of barrier

- Number and type of primary studies identifying this barrier, if provided

- Specific study designs, if stated (eg, cohort, cross sectional, comparison).

- Description of evidence of association with vaccination behaviour, including direction of association (if reported).

\section{Quality appraisal}

We used a modified version of the ROBIS (Risk of Bias in Systematic reviews) tool to identify concerns about bias in included reviews. ${ }^{11}$ We focused on the ROBIS assessments in relation to four domains: (1) study eligibility criteria, (2) identification and selection of studies, (3) data collection and study appraisal and (4) synthesis and findings. We did not assign an overall judgement of the risk of bias in each review because it was not appropriate for many of the more interpretive review types (eg, qualitative evidence syntheses). Two authors (JK and JT) independently assessed the reviews for each domain and resolved disagreements through discussion.

\section{Data synthesis}

We applied qualitative content analysis methods to inductively code the descriptions of barriers. ${ }^{12}$ We copied the sentences or phrases describing barriers from each review into a spreadsheet. Two authors (JK and JT) then independently reviewed these descriptions and condensed them into brief codes that summarised the barrier while preserving its core concept (eg, 'Concerns about vaccine safety'). We compared our codes and discussed discrepancies to arrive at an agreed phrasing and application for each code. Then, working together, we grouped similar codes together into a conceptual framework. This enabled an exploration of the relationships between barriers and characteristics of the primary studies included in each review, such as the country income level and methodology.

The degree of overlap between reviews was assessed by extracting the details for all primary studies in the original reviews and calculating the covered area and corrected covered area. ${ }^{13}$ Extracted details included author, year of publication and paper title of all primary studies. We checked for duplicates by comparing the author, year and first four words of the title using the duplicate function in Stata/IC V.16.0 (StataCorp LLC). To further identify inconsistencies, we also checked by author and title (incorrect year); year and title (incorrect author) and author and year (inconsistent title citations). When inconsistencies were noted, we reviewed the original complete title information or PubMed, as required.

\section{Patient and public involvement}

Members of the public were not involved in the review process. However, parents in Australia and New Zealand will be involved in all stages of the development and validation of the Vaccine Barriers Assessment Tool (VBAT), which will be based on the findings from this review.

\section{RESULTS}

The search retrieved 464 records. We assessed a total of 79 in full text and included 30 reviews in the final review (see PRISMA figure in online supplemental materials).

\section{Systematic review characteristics}

Reviews were published between 2005 and 2020, with the majority $(\mathrm{n}=27)$ published since 2010. A table in the online supplemental materials summarises the characteristics of included reviews and their primary studies. Fourteen reviews included both qualitative and quantitative primary studies, ${ }^{14-27}$ seven reported solely quantitative primary studies, ${ }^{28-34}$ seven reported solely qualitative primary studies ${ }^{35-41}$ and two reviews did not report the included study designs. ${ }^{42}{ }^{43}$ The reviews included 1018 primary studies from 77 countries. Twenty reviews (67\%) included studies from HIC only, with the remaining reviews featuring primary studies from LMIC $(n=6)$ or mix of country income level $(n=4)$ (figure 1$)$.

The populations of interest for all reviews were parents and/or preschool children under age 6. Most focused on 'childhood' or 'routine' vaccines in general, although some focused on a single vaccine 2529303544 or combination or multidose vaccines. ${ }^{1426}$ Some reviews had a 


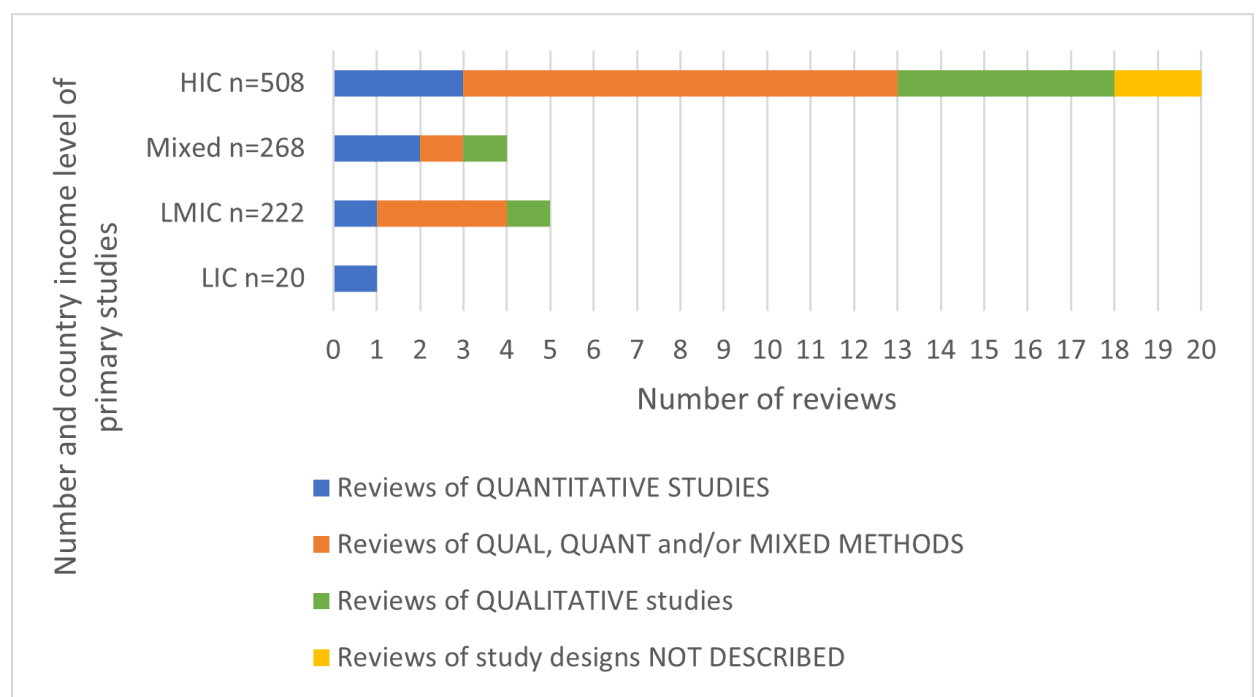

Figure 1 Methods and country income level of primary studies included in the 30 reviews (primary studies n=1018). HIC, high-income countries; LIC, low-income countries; LMIC, low-income and middle-income countries.

specific population focus, such as parents living in poor urban areas and slums, ${ }^{16}$ parents receiving care within a military health system ${ }^{42}$ or children and young people in the welfare system. ${ }^{34}$

We judged all included reviews at high or unclear concern for at least one of the ROBIS tool domains: (1) study eligibility criteria, (2) identification and selection of studies, (3) data collection and study appraisal and (4) synthesis and findings (figure 2). Many studies did not have a protocol or predefined objectives and/or they applied English language restrictions to inclusion criteria, resulting in a rating of High Risk for domain 1. Studies that were rated at High Risk for domain 2 most commonly did not include non-database sources in their searches, used very limited search terms and/or did not have two reviewers independently screening studies for inclusion. Several reviews did not provide information about how many authors extracted data and/or appraised quality of included studies, resulting in ratings of Unclear Risk for domain 3. Very few reviews had a protocol and addressed study quality in their analysis, so most were rated High Risk for domain 4. A table displaying the ratings for each domain of each review is available in the online supplemental materials.

\section{Overlap}

The reviews synthesised a total of 1018 primary studies (1022 citations). When duplicate citations were removed, there were a total of 822 unique studies. The degree of overlap in the primary studies is low, ${ }^{13}$ incorporating a covered area of $3.9 \%$ and a corrected covered area of $0.8 \%$. See online supplemental materials for a diagram illustrating the degree of overlap.

\section{Summary of findings}

In total, we extracted 573 short descriptions of parentlevel barriers. We inductively coded these barrier descriptions into a list of 64 unique barriers, thematically grouped in six overarching categories: (1) Access, (2) Clinic or Health System Barriers, (3) Concerns and Beliefs, (4) Health Perceptions and Experiences, (5) Knowledge and Information and (6) Social or Family Influence. Most reviews reported a relatively small selection of total barriers (figure 3).

Barriers in all categories were mentioned in reviews across all income settings and across the spectrum of primary study designs, although to varying degrees. The majority of reviews reported barriers from primary studies conducted in HIC countries or did not clearly

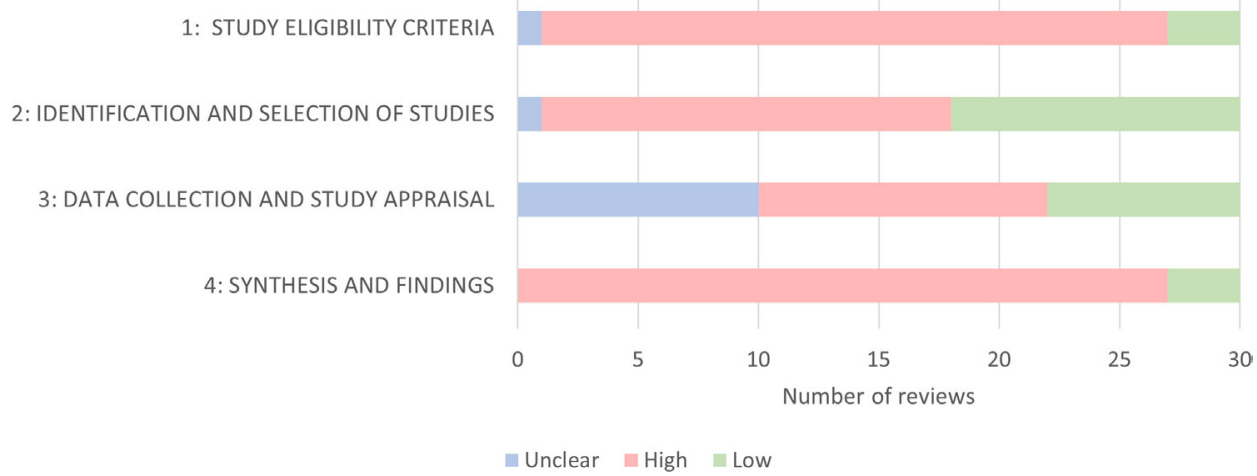

Figure 2 Graphical presentation of ROBIS assessment for included reviews. ROBIS, Risk of Bias in Systematic reviews. 


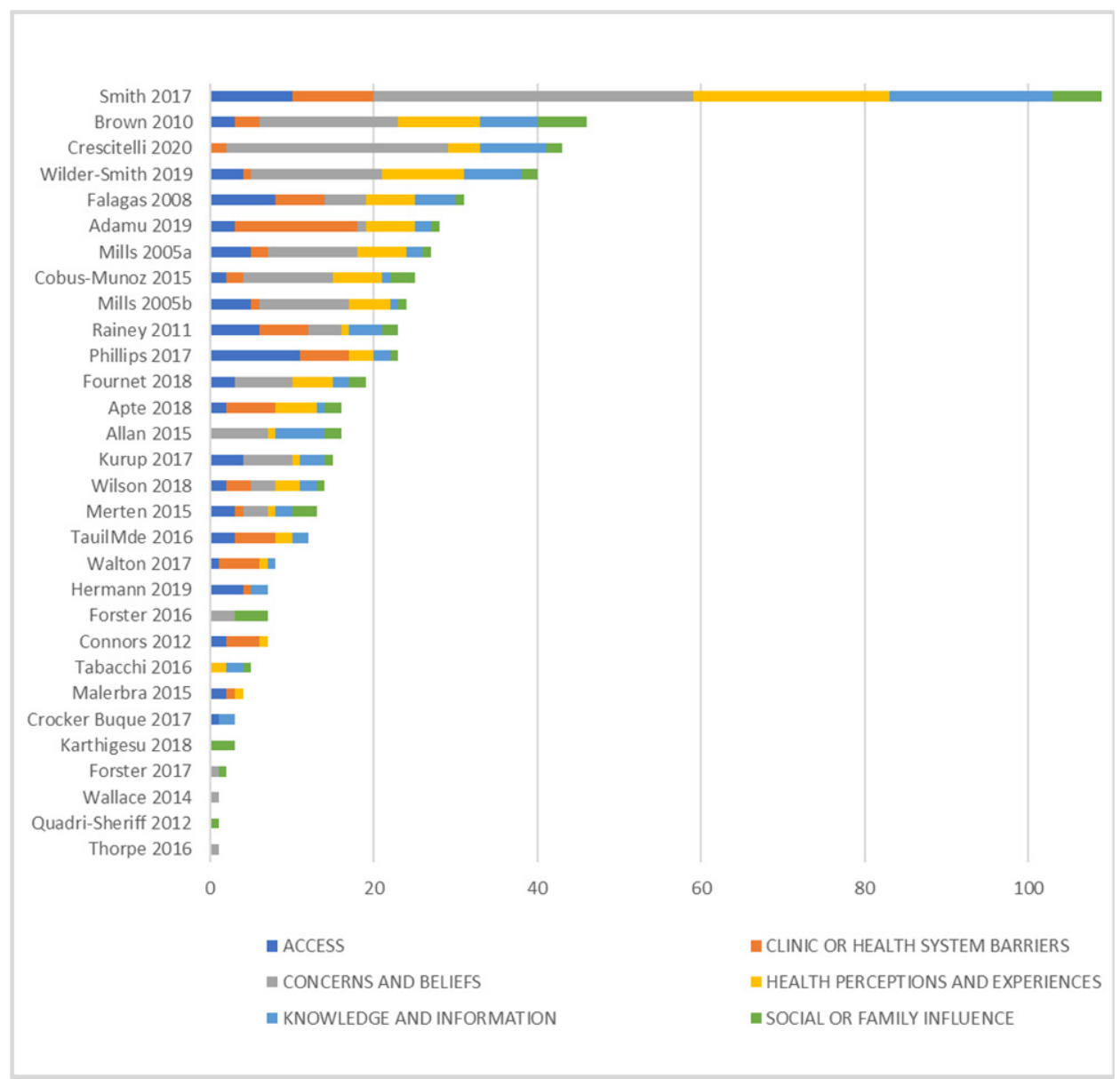

Figure 3 Included reviews by number of barrier descriptions in each category.

identify which barriers were from HIC or LMIC. The reviews which only included primary studies from LMIC settings reported barriers from all categories; there were no obvious gaps or differences in the barriers reported based on setting.

Most barriers (385/573; 67\%) were identified from reviews that incorporated primary studies with qualitative or mixed methods designs. However, because many reviews did not report which barriers came from which specific primary studies, it was not possible to identify whether there were differences in the types of barriers identified through qualitative or quantitative methods.

One of the secondary research questions was to report the evidence of each barrier's association with vaccine uptake. However, this was not possible because only one of the reviews reported this information empirically, ${ }^{14}$ and it was outside the scope of this overview to consider the data from individual primary studies.

The six categories of barriers are described below and reported in tables 1 and 2 .

\section{Access barriers}

This category included 13 individual barriers mentioned in 21 reviews (table 1 ). The most frequently mentioned access-related barrier was time constraints, followed by cost of vaccine or service delivery and transportation, distance or location. Other examples of access barriers included long clinic waiting times, childcare challenges for siblings, lack of vaccine availability and fragmented care (eg, many different providers and disconnected health records). Vulnerable populations (eg, children in the welfare system, migrants or military workers) reported specific access barriers such as impermanent residence, a lack of health insurance and social/health system exclusion or poverty.

\section{Clinic or health system barriers}

We identified 11 clinic or health system barriers in 19 reviews (table 1). The quality of provider communication/interaction was the most frequently reported barrier in this category. Other barriers in this category included providers failing to recommend vaccination, low facility quality, cultural or linguistic barriers to healthcare delivery, low provider knowledge or negative attitudes, reluctance to vaccinate and lack of relationship with the provider.

\section{Concerns or beliefs barriers}

This category included the largest number of barriers, with 16 individual barriers that related to concerns or beliefs reported in 17 reviews (table 2 ). The most frequently mentioned barriers in this category were concern about vaccine safety and lack of trust in government, vaccines or providers. Some reviews highlighted parents' 
Table 1 Access-related barriers

Access

Time constraints Explanation of barrier

Reviews reporting

Busy home life, for example, 'parents too busy.'28 the barrier $(n=21)$

Cost of vaccine or service delivery

Cost of the actual vaccines or costs related to vaccine delivery 10 (eg, appointment fees, lost parent wages due to appointment attendance).

Transportation, distance or location

Problems getting to clinic or appointment, or distance from household to health facility.

Fragmented care

Having multiple different healthcare providers, lack of

Waiting times coordinated care.

Childcare challenges

Having to wait a long time at the health facility.

5

Needing to find childcare for additional children when attending 4 vaccination appointments.

Impermanent residence

Frequent moves impacting engagement with healthcare services 4 or frequent changes of people overseeing care.

Social or health system exclusion

Receiving inadequate support from healthcare structures due to 4 poverty, discrimination and social exclusion.

Lack of vaccine availability Inability to get vaccines as needed.

4

Limited/inflexible clinic hours and inconvenient appointment 3 times.

Lack of health insurance

Financial burden of the vaccine/service for those without health insurance.

Cost of transportation

Cost of transportation to healthcare facility or indirect cost of time to take transport.

'Financial costs' and 'low access,' not further explained.

10

Access or cost: not otherwise

specified

\section{Clinic or health system}

Barrier

\section{Explanation of barrier}

Reviews reporting the barrier $(n=19)$

Poor quality provider communication/ interaction

Poor communication encounters with unpleasant, patronising, judgemental or insensitive providers.

Poor administration and recordkeeping

Lack of recall/reminder systems, incomplete or inconsistent 10 patient immunisation records, eg, 'problems with name changes and IT systems'. ${ }^{34}$

Problematic clinic practices and structure

'Reluctance to open a new vial', ${ }^{28}$ delays in clinic, disorganisation of health facilities.

Lack of vaccination recommendation

Lack of clinician recommendation/ discussion or negative recommendation.

Poor facility quality

Poor quality of health facility building and/or equipment, concern of being 'exposed to pathogens in clinics'. ${ }^{40}$

Unsupportive health system structures

Cultural or linguistic barriers to Health servic
supported.

healthcare delivery

Lack of culturally appropriate healthcare or language barriers with health workers.

Provider lack of knowledge or negative Problematic health worker attitude or knowledge. attitudes

Provider missed opportunities to Incorrectly applied contraindications, lack of screening for vaccinate vaccinations at other appointments.

Poor relationship with provider Lack of relationship or a lack of perceived clinical support from a healthcare provider.

Provider reluctance to vaccinate 
Table 2 Acceptance-related barriers

\section{Concerns and beliefs}

Barrier

Explanation of barrier

Reviews reporting the barrier $(n=19)$

Concern about vaccine safety

Lack of trust in government, vaccines or providers

Preference for natural immunity/belief in benefit of disease

Concern that vaccines compromise immune system

Alternative beliefs about health

Concern vaccines delivered too young

Belief in personal choice

Concern about combined injections

Concern about number of vaccines

Lack of self-efficacy or perceived behavioural control

Concern about autism

Conspiracy beliefs

Concern about pain

Anticipated regret

Concern about schedule

General concern (unspecified)

Health perceptions and experiences

Barrier

Perceived contraindications

Perception that disease is not severe or child is not susceptible

Allergy or adverse event experience

Perception that vaccines are not effective

Complementary and complementary medicine use

Personal objections to vaccination

Previous vaccination decision

Needle phobia

Previous/current health behaviours

\section{Knowledge and information}

\section{Barrier}

Lack of knowledge about diseases and/ or vaccines

Insufficient information

Perceived short-term and longer-term harm caused by vaccines, beliefs that 14 vaccines are unhealthy, concern over vaccine ingredients.

Mistrust of those involved in vaccine delivery and policy. Perceived influence 14 of stakeholders on decision making, eg, 'medical community does not understand adverse events', 40

Belief that natural immunity, rather than immunity from vaccines, is better. 8 Belief that 'illness strengthens child's immune system'. 31

Belief that vaccines overload the immune system or impair the body's 7 natural immunity.

Belief in immune system variation, the ability to control a child's exposure to 6 pathogens and anthroposophic ideas about disease prevention.

Belief that vaccines are provided when children are too young and not $\quad 6$ robust.

Prioritisation of parent's right to choose re: vaccination.

Belief that combination vaccines are harmful for example, 'parents perceive 5 overload of antigens'. ${ }^{18}$

Belief that children receive too many injections, too many vaccines, eg, $\quad 5$ 'multiple vaccinations are unsafe'. 31

Lack of perceived behavioural control or capacity to take their child to receive vaccines, including isolation/lack of empowerment of women.

Personal experience of autism or belief that vaccines cause autism.

Belief in conspiracy theories about vaccination.

Concern with injection site pain. if vaccine side-effects occurred.

Desire for flexibility to adapt a vaccine schedule to a child. 1

Concerns about vaccination, including parents' general worry or anxiety.

1

\section{Explanation of barrier}

Reviews reporting the barrier $(n=22)$

Perception that child has a contraindication on appointment day, eg, 10 'vaccines will be harmful if the child is sick'.15

Low perceived risk of infection or severity of illness.

Concern about potential allergy, previous traumatic or adverse vaccine experiences.

9

9

Preference for alternative healthcare for example, homeopathy.

7

Resistance, objection or disagreement with vaccines, for example, 'not

6 believing in' vaccines or 'opposition to the use of animals in vaccine development'. ${ }^{14}$

History of delayed, missed or declined vaccinations.

6

Child or parent fear of needles.

2

History of failure to engage with health services, baby health checks, eg, 'refusal of health checks'. ${ }^{34}$

2

\section{Explanation of barrier}

Reviews reporting the barrier $(n=22)$

Insufficient knowledge of what vaccines are or the importance of receiving all vaccine doses.

Insufficient quantity and quality of information. 
Table 2 Continued

\begin{tabular}{|c|c|c|}
\hline \multicolumn{3}{|l|}{ Concerns and beliefs } \\
\hline Barrier & Explanation of barrier & $\begin{array}{l}\text { Reviews reporting } \\
\text { the barrier }(n=19)\end{array}$ \\
\hline $\begin{array}{l}\text { Lack of knowledge or awareness of } \\
\text { schedule }\end{array}$ & $\begin{array}{l}\text { Insufficient knowledge about the vaccination schedule or vaccine doses, for } \\
\text { example, eligibility requirements or remembering the vaccine schedule. }\end{array}$ & 6 \\
\hline Misleading information from media & $\begin{array}{l}\text { Over-reliance on information from the media, adverse media publicity and } \\
\text { inadequate or poorly targeted mass media messaging. }\end{array}$ & 6 \\
\hline Dissatisfaction with information & Information not adequate in terms of amount, content or delivery. & 4 \\
\hline $\begin{array}{l}\text { Lack of knowledge or awareness of } \\
\text { services }\end{array}$ & Unawareness of vaccination services, clinic location or timing. & 4 \\
\hline $\begin{array}{l}\text { Lack of awareness or understanding of } \\
\text { vaccination responsibility }\end{array}$ & Uncertainty and confusion over responsibility for arranging the vaccinations. & 3 \\
\hline Forgetting that immunisation was due & Not remembering appointments or the schedule. & 3 \\
\hline Inaccurate or inappropriate information & Conflicting information or information not appropriate to education level. & 3 \\
\hline $\begin{array}{l}\text { Misleading information identified through } \\
\text { personal information seeking }\end{array}$ & $\begin{array}{l}\text { Engagement in personal research and alternate information seeking } \\
\text { behaviour, perception that 'vaccine research is vital but inadequate'. }{ }^{14}\end{array}$ & 3 \\
\hline \multicolumn{3}{|l|}{ Social or family influence } \\
\hline Barrier & Explanation of barrier & $\begin{array}{l}\text { Reviews reporting } \\
\text { the barrier }(n=22)\end{array}$ \\
\hline Social or family pressure & $\begin{array}{l}\text { Normative beliefs, subjective norms, social judgement, influence from } \\
\text { social networks or cultural pressure, family members, community members, } \\
\text { organised groups or institutions and media and the internet. }\end{array}$ & 13 \\
\hline Religious beliefs & Religious objection to vaccination. & 9 \\
\hline Lack of social responsibility & $\begin{array}{l}\text { Disinclination to vaccinate for the benefit of wider society, viewing } \\
\text { vaccinating for community protection as an unwelcome obligation. }\end{array}$ & 5 \\
\hline Family structure, roles and values & $\begin{array}{l}\text { Family roles positioning vaccination as a 'feminised task', }{ }^{39} \text { discriminatory } \\
\text { values such as a gender preference. }\end{array}$ & 2 \\
\hline Traditional or cultural beliefs & $\begin{array}{l}\text { Traditional beliefs or customs, magico-religious factors or sorcery as social } \\
\text { dimension of illness. }\end{array}$ & 2 \\
\hline
\end{tabular}

concerns that vaccines compromise the immune system, while others stated a preference for natural immunity/ benefit of disease or held specific beliefs about health related to their ability to control their child's exposure to pathogens. Other specific barriers included concerns that vaccines are delivered when children are too young and concerns about combined injections, the number of vaccines, autism, pain and the rigid vaccination schedule.

\section{Health perceptions and experiences}

This category included nine barriers mentioned in 22 reviews (table 2). While closely related to 'concerns or beliefs', barriers in this category are grounded in people's experiences and how they perceive and interpret other people's experiences related to health. Perceived contraindications were barriers when parents believed children could not be vaccinated if they had a minor non-febrile illness, such as a viral upper respiratory tract infection or 'a cold'. The perception that diseases prevented by vaccines are not serious or that children were not at risk of contracting them was another commonly reported barrier. Parents recall of vaccination experiences also contributed to vaccine decision making, such as having a known needle phobia, an allergy or adverse event experience. This was distinct from previous vaccination decision barriers which included previous vaccine delay, missed opportunity or refusal. Parents also reported specific health perceptions such as a preference for complementary and complementary medicine and personal objections to vaccination.

\section{Knowledge and information barriers}

This category included 10 barriers that were mentioned in 22 reviews (table 2 ). The most frequently mentioned barrier in this category was a lack of knowledge about diseases and/or vaccines. Closely related to this were the barriers: insufficient information, inaccurate or inappropriate information and dissatisfaction with information on vaccines generally. Vaccination schedule related barriers included confusion or lack of knowledge/ awareness of the schedule and forgetting that immunisation was due, or not remembering appointments along with knowledge/awareness of services. Inaccurate or misleading information from other sources such as media or personal information seeking were also barriers identified.

\section{Social or family influence barriers}

Despite being mentioned in 22 reviews, this category included the smallest number of distinct barriers $(n=5)$ (table 2). The most frequently reported of these, social or family pressure, focused on the perceived social approval 
of vaccination. Religious beliefs or a religious objection to vaccination, for example, 'trust in God', appeared in the second largest number of reviews. Other barriers in this category included lack of social responsibility, family structures and traditional or cultural beliefs.

\section{DISCUSSION}

This overview is the first to summarise both access and acceptance-related barriers to childhood vaccination from across a diverse range of separate systematic reviews. It is evident that the issues around childhood vaccination are multilayered and complex, with all the included reviews reporting multiple factors that require consideration by policy makers, public health services or immunisation providers. However, each review was also selective in its scope, as evidenced by the low degree of overlap between the reviews. This highlights the value of this overarching summary. Individual reviews reported on a limited range of barriers relevant to a specific population, setting or vaccine of interest. For example, most reviews and included studies were from $\mathrm{HIC}$ settings, with only one review from an LIC setting. Less than half of the included reviews reported barriers from all six of the overarching categories identified. Furthermore, only one review reported an empirical association with vaccine uptake-a critical gap in the literature.

The purpose of identifying and categorising these barriers is to bring attention to the diverse range of barriers that need to be considered when attempting to diagnose the reasons for under-vaccination in a particular population. There are many models of health behaviour that focus primarily on the internal decision-making process that leads to intention and then behaviour, such as the Health Belief Model or Theory of Planned Behaviour. ${ }^{456}$ These models incorporate psychological variables related to behaviour, but they do not address the myriad external factors that also impact behaviour in the real world. Nevertheless, they have been used to inform the development of instruments intended to measure vaccine hesitancy and predict vaccine uptake. Notable examples include the Parent Attitudes toward Childhood Vaccination scale, ${ }^{47}$ or the Vaccine Confidence Scale. ${ }^{49}$ These instruments measure the internal decision-making factors highlighted in the Health Belief Model and Theory of Planned Behaviour, such as perceived benefits of the vaccine and perceived risk of the disease. However, like the models themselves they are somewhat limited in their ability to predict actual vaccine uptake because they do not consider external factors related to access. As this overview demonstrates, there are many reasons a parent or individual might have trouble accessing vaccines, from time constraints to transportation difficulties to poor quality health facilities. By developing a comprehensive instrument that can measure both acceptance and access barriers, we can begin to target interventions effectively.

The comprehensive barrier list identified in this overview will be used in the development of such an instrument-the National Health and Medical Research Centre-funded VBAT. The VBAT will be able to diagnose both access and acceptance-related reasons for undervaccination for children under 5 years and will be validated for use in Australia and New Zealand, with possible expansion to other settings in the future. A version of the VBAT will also be developed separately for use by Indigenous and Maori parents, led by Indigenous researchers and community advisors. In this overview and in the VBAT itself, we have categorised factors that can inhibit vaccine uptake as barriers, but most barriers could also be framed as facilitators. For example, a poor clinical encounter with a judgemental provider is a barrier to vaccination, while a positive clinical encounter with an empathetic provider can drive uptake. Users of the VBAT can be flexible in how they decide to frame the key issues they identify with this tool. Our decision to choose the barrier frame is purposeful because it is typically how health policy and public health interventions are targeted-to address barriers.

Although this overview captures and organises all potential barriers to childhood vaccination described in the literature, it is only one step in the development and validation of the VBAT instrument. We also wanted to ensure that the VBAT included theoretical barriers that may not have been described in existing literature. Therefore, in addition to thematically grouping the barrier descriptions into categories in this overview, we also mapped the barrier descriptions against two theoretical models or frameworks: the COM-B (Capability, Opportunity, Motivation, Behaviour) $\operatorname{model}^{50}$ and Theoretical Domains Framework (TDF). ${ }^{51}$ These models incorporate both psychological and practical factors impacting behaviour, though they are not specific to vaccination. This additional theoretical mapping process, described elsewhere, ${ }^{52}$ allowed us to identify potential gaps where barriers might exist, despite not appearing in the literature. This process also means that the thematic groupings of barriers that we applied in this overview to facilitate comparison and summarisation do not represent the factor groupings of the eventual VBAT instrument. The final VBAT will be theoretically informed, with conceptually distinct categories. Following this comprehensive barrier identification and mapping process, we generated survey questions related to every barrier. We tested and refined these questions, along with a range of response options, through cognitive interviews with a purposive sample of potential survey participants in Australia and New Zealand. Finally, we will undertake a three-part psychometric evaluation process to reduce the items and assess dimensionality, internal consistency, construct validity, and test-retest and predictive validity.

Despite our rigorous methodological approach, this overview has several limitations. As with any overview of reviews, the evidence available for our analysis depended on the original review's focus and review author's interpretations of primary studies. On occasion, particularly for qualitative data, it was not clear whether the 
data came from the primary studies or were subject to author interpretation. We acknowledge that due to the subjective nature of thematic coding, some barriers and categories in our conceptual framework may have been grouped differently by other authors. The additional mapping to theoretical models, described above, helps address this potential bias. Finally, we acknowledge that quantitative frequency counts (ie, the number of reviews contributing data on each barrier) are not traditionally compatible with qualitative coding. However, we believe these frequency counts support our aim to describe the range of review evidence on this topic and highlight the most frequently reported barriers in the existing literature. The frequency of reporting of different barriers should not be assumed to be reflective of the importance or prevalence of this barrier. In primary quantitative survey studies in particular, participants may only be able to select from a predefined list of barriers. Qualitative data are less constrained by such predefined limits, and therefore a strength of this review is its inclusion of reviews of mixed-methods studies. Finally, $67 \%$ of the included reviews were from HIC only, with only six reviews from LMIC settings. While this means more individual barrier descriptions were identified from HIC settings, every barrier category included data from LMIC settings as well.

\section{CONCLUSIONS}

This overview of systematic reviews provides a comprehensive summary of barriers to childhood vaccination in different healthcare settings, from the parent perspective. The included reviews identified both access and acceptance-related barriers to vaccination, predominantly from HIC settings, with more data needed from middle-income and low-income settings. These findings will inform the development of a tool to accurately measure both access and acceptance barriers to childhood vaccination and identify the causes of undervaccination in preschool-aged children. This tool can be used to support provider-parent vaccine discussions and enable development, targeting and evaluation of costeffective interventions to increase vaccine uptake.

\section{Author affiliations \\ ${ }^{1}$ Vaccine Uptake Group, Murdoch Childrens Research Institute, Parkville, Victoria, Australia \\ ${ }^{2}$ Faculty of Medicine Dentistry and Health Sciences, The University of Melbourne, Melbourne, Victoria, Australia \\ ${ }^{3}$ Faculty of Medicine and Health, The University of Sydney School of Public Health, Sydney, New South Wales, Australia \\ ${ }^{4}$ Health Protection, Hunter New England Health, Wallsend, New South Wales, Australia \\ ${ }^{5}$ School of Medicine and Public Health, The University of Newcastle, Callaghan, New South Wales, Australia}

Acknowledgements We would like to thank Michael Fajardo and Keana Loschiavo for their help screening papers and extracting data in early stages of this review.

Contributors All authors were involved in review conceptualisation and protocol development. JK and JT conducted searches, extracted data, critically appraised included reviews and categorised barriers for analysis, with additional input from $\mathrm{CB}$ and MD. All authors contributed to drafting the manuscript.

Funding This review was funded by an Australian National Health and Medical Research Council grant (GNT 1164200).

Competing interests None declared.

Patient consent for publication Not required.

Provenance and peer review Not commissioned; externally peer reviewed.

Data availability statement All data relevant to the study are included in the article or uploaded as supplementary information. Not applicable.

Supplemental material This content has been supplied by the author(s). It has not been vetted by BMJ Publishing Group Limited (BMJ) and may not have been peer-reviewed. Any opinions or recommendations discussed are solely those of the author(s) and are not endorsed by BMJ. BMJ disclaims all liability and responsibility arising from any reliance placed on the content. Where the content includes any translated material, BMJ does not warrant the accuracy and reliability of the translations (including but not limited to local regulations, clinical guidelines, terminology, drug names and drug dosages), and is not responsible for any error and/or omissions arising from translation and adaptation or otherwise.

Open access This is an open access article distributed in accordance with the Creative Commons Attribution Non Commercial (CC BY-NC 4.0) license, which permits others to distribute, remix, adapt, build upon this work non-commercially, and license their derivative works on different terms, provided the original work is properly cited, appropriate credit is given, any changes made indicated, and the use is non-commercial. See: http://creativecommons.org/licenses/by-nc/4.0/.

\section{REFERENCES}

1 Durrheim DN, Andrus JK, Tabassum S, et al. A dangerous measles future looms beyond the COVID-19 pandemic. Nat Med 2021;27:360-1.

2 Harris RC, Chen Y, Côte P, et al. Impact of COVID-19 on routine immunisation in south-east Asia and Western Pacific: disruptions and solutions. Lancet Reg Health West Pac 2021;10:100140.

3 World Health Organization. Ten threats to global health in 2019, 2019. Available: https://www.who.int/emergencies/ten-threats-toglobal-health-in-2019

4 Nour TY, Farah AM, Ali OM, et al. Immunization coverage in Ethiopia among 12-23 month old children: systematic review and metaanalysis. BMC Public Health 2020;20:1134.

5 de Figueiredo A, Simas C, Karafillakis E, et al. Mapping global trends in vaccine confidence and investigating barriers to vaccine uptake: a large-scale retrospective temporal modelling study. Lancet 2020;396:898-908.

6 Mahase E. Vaccination uptake: access is still biggest barrier, experts warn. BMJ 2019;366:I5576.

7 Aromataris E, Fernandez R, Godfrey C. Chapter 10: Umbrella Reviews. In: JBI manual for evidence synthesis. JBI, 2020. https:// synthesismanual.jbi.global

8 Kaufman J, Tuckerman J, Durrheim D, et al, 2019Barriers to uptake of childhood vaccination: protocol for a review of systematic reviews. Available: https://melbourne.figshare.com/articles/preprint/ Barriers_to_uptake_of_childhood_vaccination_protocol_for_a_ review_of_systematic_reviews/11345057

9 Page MJ, McKenzie JE, Bossuyt PM, et al. The PRISMA 2020 statement: an updated guideline for reporting systematic reviews. BMJ 2021;372:n71.

10 Veritas Health Innovation. Covidence systematic review software. Melbourne, Australia. Available: www.covidence.org

11 Whiting P, Savović J, Higgins JPT, et al. ROBIS: a new tool to assess risk of bias in systematic reviews was developed. J Clin Epidemiol 2016;69:225-34.

12 Graneheim UH, Lundman B. Qualitative content analysis in nursing research: concepts, procedures and measures to achieve trustworthiness. Nurse Educ Today 2004;24:105-12.

13 Pieper D, Antoine S-L, Mathes T, et al. Systematic review finds overlapping reviews were not mentioned in every other overview. $J$ Clin Epidemiol 2014;67:368-75.

14 Brown KF, Kroll JS, Hudson MJ, et al. Factors underlying parental decisions about combination childhood vaccinations including MMR: a systematic review. Vaccine 2010;28:4235-48.

15 Cobos Muñoz D, Monzón Llamas L, Bosch-Capblanch X. Exposing concerns about vaccination in low- and middle-income countries: a systematic review. Int J Public Health 2015;60:767-80. 
16 Crocker-Buque T, Mindra G, Duncan R, et al. Immunization, urbanization and slums - a systematic review of factors and interventions. BMC Public Health 2017;17:556.

17 Falagas ME, Zarkadoulia E. Factors associated with suboptimal compliance to vaccinations in children in developed countries: a systematic review. Curr Med Res Opin 2008;24:1719-41.

18 Fournet N, Mollema L, Ruijs WL, et al. Under-vaccinated groups in Europe and their beliefs, attitudes and reasons for non-vaccination; two systematic reviews. BMC Public Health 2018;18:196.

19 Hermann JS, Featherstone RM, Russell ML, et al. Immunization coverage of children in care of the child welfare system in high-income countries: a systematic review. Am J Prev Med 2019;56:e55-63.

20 Kurup L, Shorey S, Wang W, et al. An integrative review on parents' perceptions of their children's vaccinations. J Child Health Care 2017;21:343-52.

21 Mills EJ, Montori VM, Ross CP, et al. Systematically reviewing qualitative studies complements survey design: an exploratory study of barriers to paediatric immunisations. J Clin Epidemiol 2005;58:1101-8.

22 Phillips DE, Dieleman JL, Lim SS, et al. Determinants of effective vaccine coverage in low and middle-income countries: a systematic review and interpretive synthesis. BMC Health Serv Res 2017; 17:681.

23 Quadri-Sheriff M, Hendrix KS, Downs SM, et al. The role of herd immunity in parents' decision to vaccinate children: a systematic review. Pediatrics 2012;130:522-30.

24 Rainey JJ, Watkins M, Ryman TK, et al. Reasons related to nonvaccination and under-vaccination of children in low and middle income countries: findings from a systematic review of the published literature, 1999-2009. Vaccine 2011;29:8215-21.

25 Tabacchi G, Costantino C, Napoli G, et al. Determinants of European parents' decision on the vaccination of their children against measles, mumps and rubella: a systematic review and metaanalysis. Hum Vaccin Immunother 2016;12:1-15.

26 Wallace AS, Mantel C, Mayers G, et al. Experiences with provider and parental attitudes and practices regarding the administration of multiple injections during infant vaccination visits: lessons for vaccine introduction. Vaccine 2014;32:5301-10.

27 Wilder-Smith AB, Qureshi K. Resurgence of measles in Europe: a systematic review on parental attitudes and beliefs of measles vaccine. J Epidemiol Glob Health 2020;10:46-58.

28 Adamu AA, Sarki AM, Uthman OA, et al. Prevalence and dynamics of missed opportunities for vaccination among children in Africa: applying systems thinking in a systematic review and meta-analysis of observational studies. Expert Rev Vaccines 2019;18:547-58.

29 Apte A, Roy S, Bavdekar A, et al. Facilitators and barriers for use of rotavirus vaccine amongst various stakeholders and its implications for Indian context - A systematic review. Hum Vaccin Immunother 2018;14:1-8.

30 Malerba V, Costantino C, Napoli G, et al. Antimeningococcal and antipneumococcal vaccination determinants: a European systematic literature review. Epidemiol Prev 2015;39:59-64.

31 Smith LE, Amlôt R, Weinman J, et al. A systematic review of factors affecting vaccine uptake in young children. Vaccine 2017;35:6059-69.

32 Tauil MdeC, Sato APS, Waldman EA. Factors associated with incomplete or delayed vaccination across countries: a systematic review. Vaccine 2016;34:2635-43.

33 Thorpe S, VanderEnde K, Peters C, et al. The influence of women's Empowerment on child immunization coverage in low, Lower-
Middle, and Upper-Middle income countries: a systematic review of the literature. Matern Child Health J 2016;20:172-86.

34 Walton S, Bedford $\mathrm{H}$. Immunization of looked-after children and young people: a review of the literature. Child Care Health Dev 2017;43:463-80.

35 Allan N, Harden J. Parental decision-making in uptake of the MMR vaccination: a systematic review of qualitative literature. J Public Health 2015;37:678-87.

36 Díaz Crescitelli ME, Ghirotto L, Sisson H, et al. A meta-synthesis study of the key elements involved in childhood vaccine hesitancy. Public Health 2020;180:38-45.

37 Forster AS, Rockliffe L, Chorley AJ, et al. Ethnicity-specific factors influencing childhood immunisation decisions among black and Asian minority ethnic groups in the UK: a systematic review of qualitative research. J Epidemiol Community Health 2017;71:544-9.

38 Forster AS, Rockliffe L, Chorley AJ, et al. A qualitative systematic review of factors influencing parents' vaccination decision-making in the United Kingdom. SSM Popul Health 2016;2:603-12.

39 Merten S, Martin Hilber A, Biaggi C, et al. Gender determinants of vaccination status in children: evidence from a Meta-Ethnographic systematic review. PLoS One 2015;10:e0135222.

40 Mills E, Jadad AR, Ross C, et al. Systematic review of qualitative studies exploring parental beliefs and attitudes toward childhood vaccination identifies common barriers to vaccination. J Clin Epidemiol 2005;58:1081-8.

41 Wilson L, Rubens-Augustson T, Murphy M, et al. Barriers to immunization among newcomers: a systematic review. Vaccine 2018;36:1055-62.

42 Connors J, Arushanyan E, Bellanca G, et al. A description of barriers and facilitators to childhood vaccinations in the military health system. J Am Acad Nurse Pract 2012;24:716-25.

43 Karthigesu SP, Chisholm JS, Coall DA. Do grandparents influence parents' decision to vaccinate their children? A systematic review. Vaccine 2018:36:7456-62.

44 Wilder-Smith AB, Qureshi K. Resurgence of measles in Europe: a systematic review on parental attitudes and beliefs of measles vaccine. J Epidemiol Glob Health 2020;10:46-58.

45 Ajzen I. The theory of planned behavior. Organ Behav Hum Decis Process 1991;50:179-211.

46 Rosenstock IM. The health belief model and preventive health behavior. Health Educ Monogr 1974;2:354-86.

47 Opel DJ, Mangione-Smith R, Taylor JA, et al. Development of a survey to identify vaccine-hesitant parents: the parent attitudes about childhood vaccines survey. Hum Vaccin 2011;7:419-25.

48 Opel DJ, Taylor JA, Zhou C, et al. The relationship between parent attitudes about childhood vaccines survey scores and future child immunization status: a validation study. JAMA Pediatr 2013;167:1065-71.

49 Gilkey MB, Magnus BE, Reiter PL, et al. The vaccination confidence scale: a brief measure of parents' vaccination beliefs. Vaccine 2014;32:6259-65.

50 Michie S, van Stralen MM, West R. The behaviour change wheel: a new method for characterising and designing behaviour change interventions. Implement Sci 2011;6:42

51 Cane J, O'Connor D, Michie S. Validation of the theoretical domains framework for use in behaviour change and implementation research. Implement Sci 2012;7:37.

52 Bonner C, Tuckerman J, Kaufman J, et al. Comparing inductive and deductive analysis techniques to understand health service implementation problems: a case study of childhood vaccination barriers. Implementation Science 2021;2. 